\title{
Cross sections of nuclear isomers from the interaction of protons with the thin thorium target
}

\author{
R.R. Holomb ${ }^{1,2, *}$, S.A. Tari ${ }^{2}$, K. Katovsky ${ }^{1}$, I. Haysak ${ }^{2}$, J. Adam ${ }^{3,4}$, V.B. Brudanin ${ }^{3}$, \\ $R$. Vespalec ${ }^{4}, J$. Vrzalova ${ }^{3,4}, L$. Zavorka ${ }^{3}, M$. Zeman $^{1,3}, D . V$. Karaivanov $^{3,5}, A . A$. Solnyshkin ${ }^{3}$, \\ D.V. Philosophov ${ }^{3}, J$. Khushvaktov ${ }^{3}$, and V.M. Tsupko-Sitnikov ${ }^{3}$ \\ ${ }^{1}$ Technological University of Brno, Brno, Czech Republic \\ ${ }^{2}$ Uzhgorod National University, Uzhgorod, Ukraine \\ ${ }^{3}$ Joint Institute for Nuclear Research, Dubna, Russia \\ ${ }^{4}$ Institute of Nuclear Physics, Czech Academy of Sciences, Rez, Czech Republic \\ ${ }^{5}$ Institute for Nuclear Research and Nuclear Energy, Sofia, Bulgaria
}

\begin{abstract}
The paper shows the results of experimental gamma spectra obtained with a thorium ${ }^{232} \mathrm{Th}$ target and an aluminum collector irradiated at the JINR Synchrocyclotron with the internal beam of energies of 100 and $600 \mathrm{MeV}$. For ${ }^{232} \mathrm{Th}$ there were identified 258 and 222 gamma lines that belong to 45 and 55 nuclides, respectively. For $\mathrm{Al}-238,330$ lines and 81, 119 nuclides, respectively. The cross sections of fragmentation of the ${ }^{232} \mathrm{Th}$ and $\mathrm{Al}$ nuclei under the interaction with protons 100 and $600 \mathrm{MeV}$ was determined. A comparison of the obtained cross sections of the reaction with theoretical calculations was performed.
\end{abstract}

\section{Introduction}

The interest of the world scientific community in the research of this kind is primarily associated with the problem of transmutation of the long-lived radioactive waste and the creation of subcritical nuclear power plants with the uranium-thorium cycle controlled by Accelerator Driven Subcritical Systems (ADS). These installations may also partially take on the function of the radioactive waste disposal. Also of great interest is the ability to use ${ }^{238} \mathrm{U}$ and ${ }^{232} \mathrm{Th}$ as nuclear fuel since these isotopes are more common in nature than ${ }^{235} \mathrm{U}$.

The purpose is to study the process of separation of ${ }^{232} \mathrm{Th}$ protons.

The task is processing the experimental $\gamma$-spectrum experiment performed on the Synchrocyclotron (Phasotron) at JINR, Dubna.

The object of the study is the ${ }^{232}$ Th nucleus.

The subject of the research is the reaction of fission and fragmentation of the ${ }^{232} \mathrm{Th}$ nucleus under the interaction with protons.

The research methods are the $\gamma$-spectroscopy of the irradiated target and the collector of the fragments. For theoretical calculations a Monte Carlo method using the MCNP 61.0 program with the CEM model was used.

\footnotetext{
*e-mail: xholom05@stud.feec.vutbr.cz
} 


\subsection{JINR}

In Russia, a number of studies on the irradiation of small targets on direct proton beams have been performed in ITEF. These works studied the transmutation of the long-lived waste, primarily ${ }^{129} \mathrm{I}$ and younger actinides ${ }^{237} \mathrm{Kr},{ }^{238} \mathrm{U},{ }^{239} \mathrm{Pu},{ }^{241} \mathrm{Am}$, have been conducted within the framework of a broad international collaborative work on the subcritical installation "Energy plus transmutation", "QUINTA" and "BURAN" on the Phasotron beam LFBE [1,2].

\subsection{MYRRHA}

A multipurpose hybrid research reactor for application in the field of high technology is the setup using fast neutrons with the lead-bismuth coolant and heat capacity of 50-100 MW. It is designed as a system using an accelerator to operate in subcritical and critical modes. The reactor is scheduled to be commissioned in 2023 [3].

\subsection{Installation of neutron generation in KIPT}

The US government supports the development, construction and operation of an ADS system (neutron source research facility, KHPTI) at the Kharkiv Institute of Physics and Technology (KPI) of Ukraine under the Russian Research Reactor Fuel Return (RRRFR) program of the United States Department of Energy.

This device consists of a sub-critical accelerator system that uses the low-enriched uranium oxide with a cooling fluid (water) and the beryllium-carbon reflector. An electron accelerator is used to create a neutron source used by a subcritical device to function. The target of this installation is in the middle of the tungsten plate or the natural uranium used to create neutrons that cool water. Tungsten or uranium is the target material for generating neutrons. Water, like the coolant and the aluminum alloy structure, are used as a target. The target configuration is designed to place a beam square profile and hexagonal fuel geometry. The power of the accelerator beam is $100 \mathrm{~kW}$ from $100 \mathrm{MeV}$ electrons [4].

\section{Experiment}

The experiment was preformed on the Phasotron in DLNP, JINR (Fig. 1). The maximum energy of accelerated protons at Phasotron is $660 \mathrm{MeV}$.

The main parameters of the Phasotron are:

1. Energy of accelerated protons $-\mathrm{T}_{r}=(659 \pm 6) \mathrm{MeV}$.

2. Energy dispersion $-\mathrm{T}_{e}=(3.1 \pm 0.8) \mathrm{MeV}$.

3. Frequency of proton acceleration cycles (modulation frequency) $-250 \mathrm{~Hz}$.

Emittance at the boundary of the scattered magnetic field of the Phasotron:

1. Horizontal $\mathrm{e}_{x}=(5,1 \pm 2,3) \mathrm{cm} * \operatorname{mrad}$.

2. Vertical $\mathrm{e}_{y}=(3.4 \pm 1.4) \mathrm{cm}^{*} \mathrm{mrad}$.

3. Intensity of the extracted proton beam mode "fast" output (pulse duration $30 \mathrm{mks}$ ) (22,5) mkA.

4. Intensity of the extracted proton beam in the "slow" output mode (beam extended in time for $85 \%$ of the modulation period $(\sim 4 \mathrm{mc})$ ) (1.6-2.0) $\mathrm{mkA}$.

5. Extracted proton beam has a microstructure - bunches of particles with a duration of about $10 \mathrm{~ns}$ follow with an interval of about $70 \mathrm{~ns}$. 


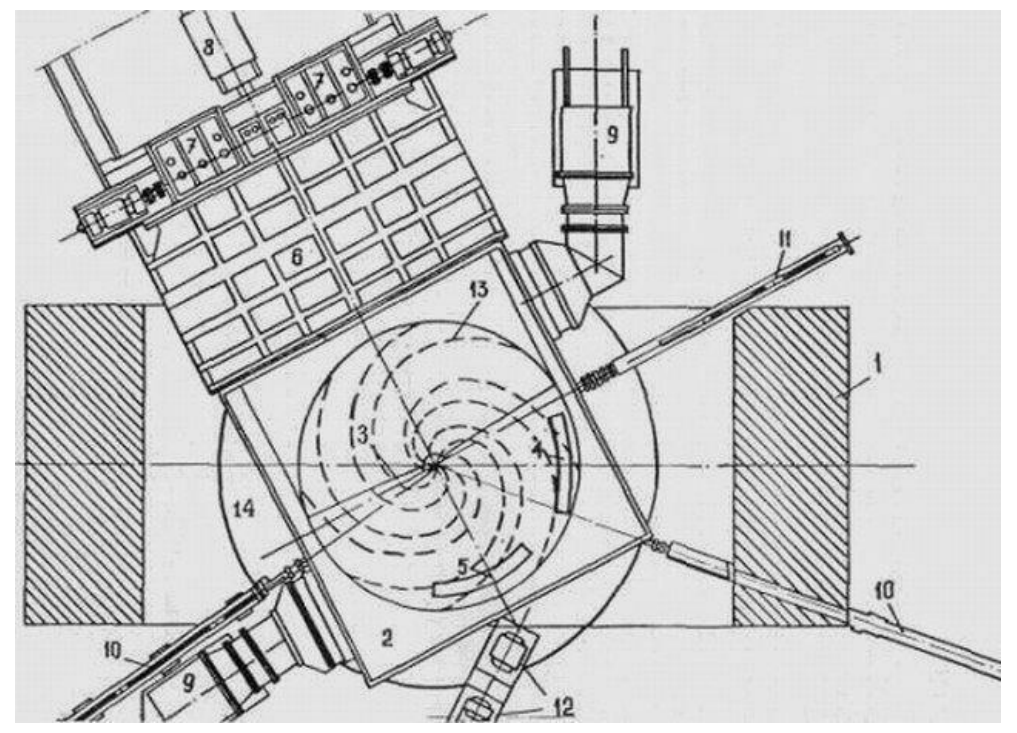

Figure 1: Schema of the Phasotron in DLNP, JINR

1 - magnet housing; 2 - vacuum chamber; 3 - duant; 4 - output channel; 5 - SI-electrode for beam stretching; 6 - intermediate chamber; 7 - variator; 8 - HF generator; 9 - vacuum pumps; 10 - samplers; 11 - ion source rod; 12 - the first magnetic elements of the proton path; 13 - spiral shimmy for the spatial variation of the magnetic field; 14 - magnet excitation winding

The samples, using a special device, were placed in the accelerator chamber at a radius corresponding to the energy of protons 100 and $600 \mathrm{MeV}$ at the current of $0.3 \mu \mathrm{A}$. The position of the 100 and $600 \mathrm{MeV}$ beams was determined by placing an aluminum foil inside the accelerator and perpendicular to the proton beam and evaluating the activity of the foil. For the target, foils of ${ }^{232} \mathrm{Th}$ were used with a thickness of 100 microns and a weight of $149.5 \mathrm{mg}$ placed between two Al foils with thickness of $50 \mathrm{mkm}$. The foil area was $1.5 \mathrm{~cm}^{2}$. The target was placed in such way that the proton beam was hitting the edge of the target as it shows on Fig. 2. The dimensions of the beam in the cross section were $\Delta \mathrm{X}=2.5 \mathrm{~cm}$ and $\Delta \mathrm{Y}=2.6 \mathrm{~cm}$. To determine the integral flow of protons falling on the ${ }^{232} \mathrm{Th}$ samples, an activation method of ${ }^{27} \mathrm{Al}$ with ${ }^{24} \mathrm{Na}$ was used. For this purpose, two

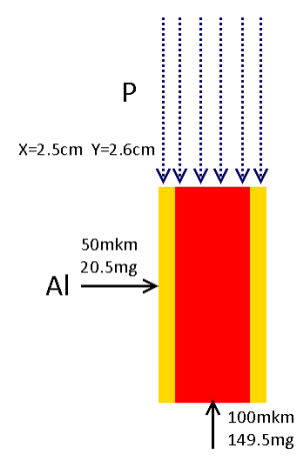

Th 232

Figure 2: ${ }^{232}$ Th target sides of the ${ }^{232} \mathrm{Th}$ were placed close to the ${ }^{27} \mathrm{Al}$ foil $(50 \mu \mathrm{m})$. As a result, for each of the irradiations, an integral proton flow on samples was determined, which was $7.64 \cdot 10^{12}$.

After irradiation, the samples were removed from the chamber of the Phasotron and moved to the spectroscopic complex YSNAPP-2, which separately measured the spectra of $\gamma$ radiation of the ${ }^{232} \mathrm{Th}$ and ${ }^{27} \mathrm{Al}$ foils using the HPGe-detector of the CANBERRA company with an efficiency of $18 \%$ and a resolution of $1.9 \mathrm{keV}$ in the line $1332 \mathrm{keV}{ }^{60} \mathrm{Co}$. The measurement time was $1 \mathrm{~m}, 2 \mathrm{~m}, 4 \mathrm{~m}, 8 \mathrm{~m}, 16 \mathrm{~m}, 30 \mathrm{~m}, 1 \mathrm{~h}, 2 \mathrm{~h}, 4 \mathrm{~h}, 6 \mathrm{~h}, 12 \mathrm{~h}, 1 \mathrm{~d}, 2 \mathrm{~d}$ [5]. 


\section{Processing of gamma spectra}

The processing of the gamma spectra was carried out using the DEIMOS32 program to find positions of peaks, their areas and other parameters. The identification of the nuclei formed in ${ }^{232} \mathrm{Th}$ samples as a result of nuclear reactions with protons was carried out using a set of scripts based on the Ruby programming language (AttCor , EffCor, MidLit5, NonLin64, PureGam, SepDepe, SigmaJ7, TimeConst, TrueConic, TransCs9). After that, the cross sections of the obtained isotopes were compared with data from the Los Alamos National Laboratory under the program MCNP6 1.0 (the database of theoretically calculated cross sections of isotopes CEM100.asc) [6], [7].

\section{Results}

\section{$4.1{ }^{232} \mathrm{Th}$}

For $100 \mathrm{MeV}, 45$ nuclides were identified. The identified nuclides have a mass number in the range of 71-224 with spaces in the range of values $100-110$ and 150-223. Fig. 1 shows the dependence of the cross section of the fragmentation of nuclei on the mass number A. The intervals of the mass number of nuclides correspond to the intervals of the atomic number $\mathrm{Z}$ 44, 45 (Fig. 3a) and 61-88 (Fig. 3b).

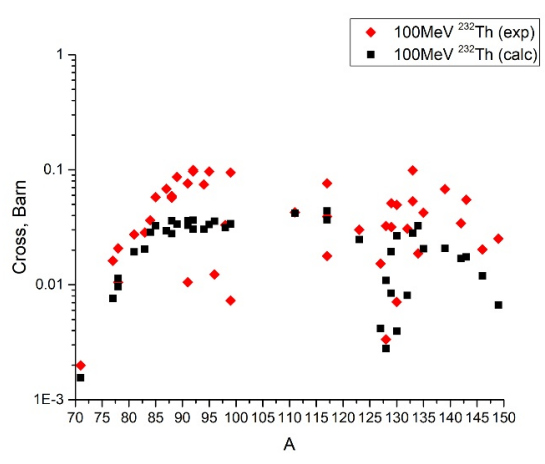

(a)

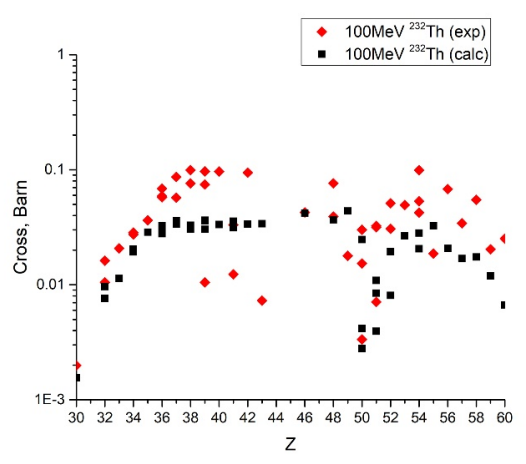

(b)

Figure 3: Graph of the cross sections for thorium irradiated by $100 \mathrm{MeV}$ protons

As a result of processing of gamma spectra measured for the irradiated thorium target, 222 gamma lines were identified. The comparison of the obtained data with published results allowed identification of 55 nuclides by energy and half-life. The identified nuclides have a mass number in the 68-211 range with spaces in the range of values 134-142 and 143-186. The spacing of the mass number of nuclides corresponds to spaces of the serial number $\mathrm{Z}$ of 54-57 (Fig. 4a) and 58-77 (Fig. 4b). The sections of fragmentation of the 232Th nucleus under the action of $600 \mathrm{MeV}$ of protons were determined, respectively. 


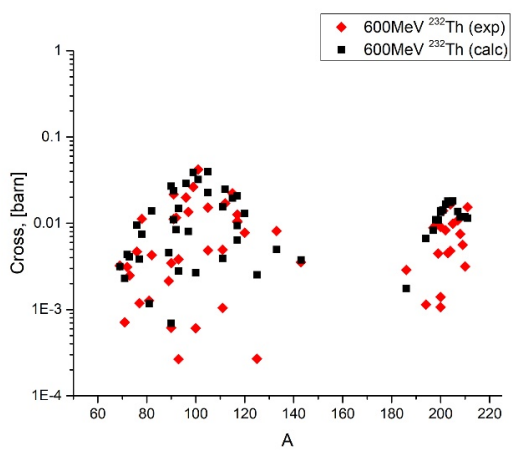

(a)

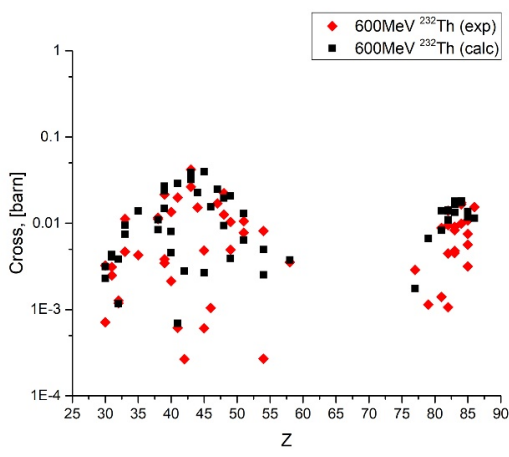

(b)

Figure 4: Graph of the cross sections for thorium irradiated by $600 \mathrm{MeV}$ protons

\section{$4.2 \mathrm{Al}$}

The 81 nuclides were identified. The identified nuclides have a mass number in the range of 24-233 with spaces in the range of values $Z$ and A 63-71 (Fig. 5a) and 150-171 (Fig. 5b), respectively. Fig. 5 shows the dependence of the fragmentation cross section of nuclei on the mass number A. Fig. 6 shows the dependence of the fragmentation cross section of nuclei on the serial number $\mathrm{Z}$.

The 119 nuclides were identified. The identified nuclides have a mass number in the range of 7-237 with spaces in the range of values Z and A 58-69 and 143-167, respectively. Fig. 6a shows the dependence of the fragmentation cross section of nuclei on the mass number A. Fig. $6 \mathrm{~b}$ shows the dependence of the fragmentation cross section of nuclei on the serial number $\mathrm{Z}$.

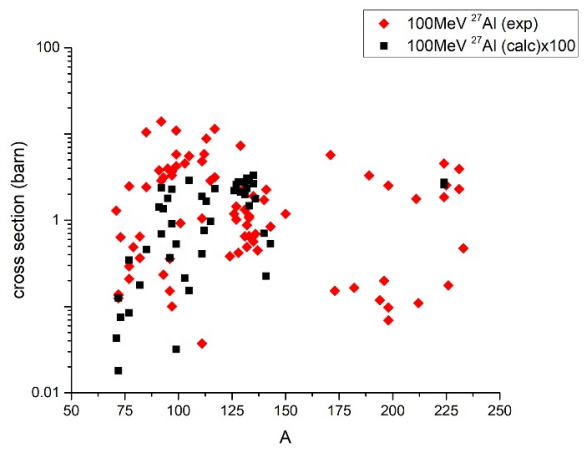

(a)

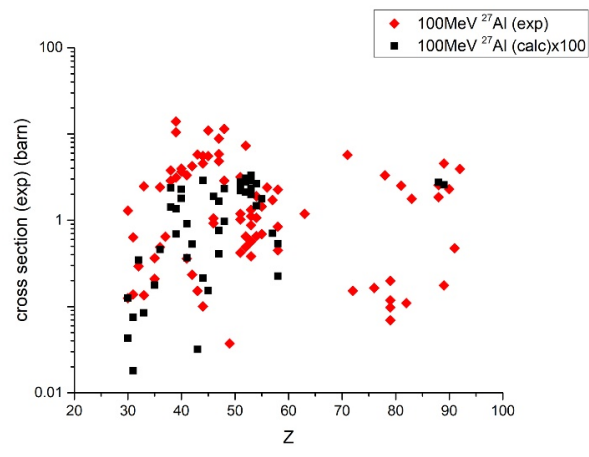

(b)

Figure 5: Graph of the cross sections for aluminum irradiated by $100 \mathrm{MeV}$ protons 


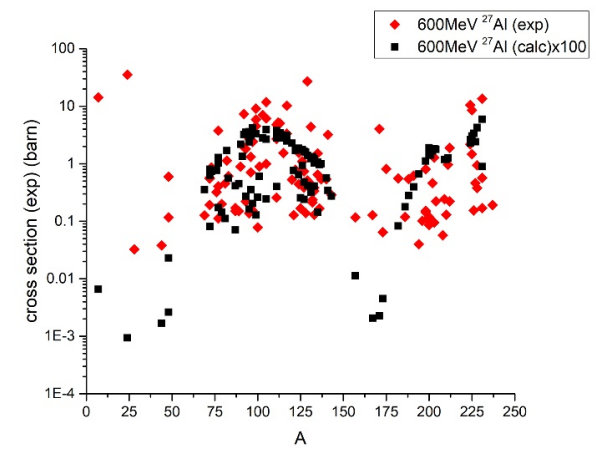

(a)

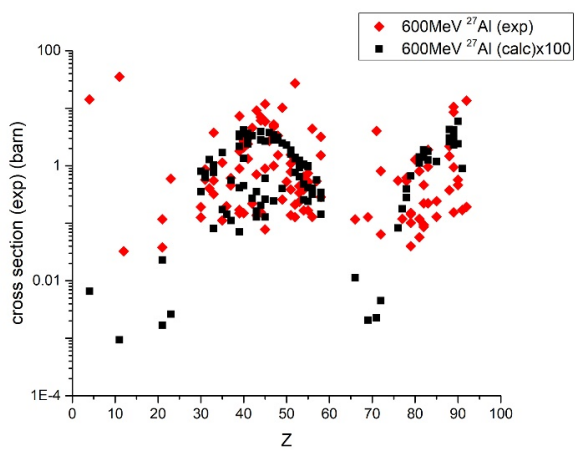

(b)

Figure 6: Graph of the cross sections for aluminum irradiated by $600 \mathrm{MeV}$ protons

\section{Conclusion}

The experimental data on the fragmentation of the ${ }^{232} \mathrm{Th}$ nucleus under the influence of protons in the energy of 100 and $600 \mathrm{MeV}$ have been processed. The experiment is performed at the Phasotron in JINR, Dubna. The sections of fragmentation of the ${ }^{232}$ Th nucleus have been obtained depending on the charge and mass number of the reaction fragments. For the $100 \mathrm{MeV} 232 \mathrm{Th}$ targets the 258 gamma lines that belong to 45 nuclides and 222 belong to 55 nuclides in case of $600 \mathrm{MeV}$ have been identified. For the Al collector, the 238 gamma lines belonging to 81 nuclides in case of $100 \mathrm{MeV}$ and 330 lines for 119 nuclides in case of $600 \mathrm{MeV}$, respectively, have been identified. The incommensurability of the number of lines for the target and the collector can be explained by the different distribution of fragments by kinetic energy. For the mass and charge spectra, the separation and fragmentation reactions are clearly divided.

\section{References}

[1] J.H. Khushvaktov, J. Adam, A.A. Baldin, W.I. Furman, S.A. Gustov, Yu.V. Kish, A.A. Solnyshkin, V.I. Stegailov, J. Svoboda, P. Tichy, V.M. Tsoupko-Sitnikov, S.I. Tyutyunnikov, R. Vespalec, J. Vrzalova, V. Wagner, B.S. Yuldashev, L. Zavorka, M. Zeman, Applied Radiation and Isotopes 137, 102-107 (2018)

[2] I. Adam, V. Wagner, I. Vrzalova, L. Zaworka, M. G. Kadykov, V.S. Pronsky, A.A. Solnyshkin, V.I. Steigailov, M. Suhopar, S.I. Tyutyunnikov, V.I. Furman, Zh. Khushvaktov, V.M. Tsupko-Sitnikov, V.V. Chilap, Applied Radiation and Isotopes 107, 225-233 (2015)

[3] http://www.atomic-energy.ru/news/2012/01/16/30122

[4] M.I. Ayzatskiy, B.V. Borts, A.N. Vodin, P.A. Demchenko, A.Yu. Zelinskiy, I.M. Karnaukhov, V.A. Kushnir, V.V. Mitrochenko, A.O. Mytsykov, I.M. Neklyudov, S.N. Oleynik, F.A. Peev, G.D. Pugachev, S.A. Soldatov, I.V. Ushakov, Y .Gohar, I. Bolshinsky, Ya.L. Chi, S.L. Pei, S.H. Wang, W.B. Liu, Problems of atomic science and technology 79, 3-9 (2012)

[5] R. Holomb, K. Katovský, I Adam, M. Zeman, I. Haysak, A. Solnyshkin, J. Khushvaktov, R. Vespalets, in Proccedings of the 2018 19th International Scientific Conference on Electric Power Engineering (EPE) (Brno: VUT, 2018) 527-531

[6] S.G. Mashnik and A.J. Sierk, LANL Report LA-R-12-01364 (2012)

[7] F.B. Brown, LANL Report LA-UR-13-23040 (2013) 